BI-TH 2001/13

MPI-PhT/2001-23, July 16, 2001

\title{
Scaling in $\gamma^{*} p$ total cross sections, saturation and the gluon density *
}

\author{
Dieter Schildknecht \\ Fakultät für Physik, Universität Bielefeld \\ D-33501 Bielefeld, Germany** \\ and MPI für Physik, München, Germany \\ Bernd Surrow \\ DESY, 22607 Hamburg, Germany \\ and \\ Mikhail Tentyukov*** \\ Fakultät für Physik, Universität Bielefeld \\ D-33501 Bielefeld, Germany
}

\begin{abstract}
Including the new HERA data, the $\gamma^{*} p$ total cross section is analysed in the generalized vector dominance/colour-dipole picture (GVD/CDP) that contains scaling in $\eta=\left(Q^{2}+m_{0}^{2}\right) / \Lambda^{2}\left(W^{2}\right)$, where $\Lambda^{2}\left(W^{2}\right)$ is an increasing function of $W^{2}$. At any $Q^{2}$, for $W^{2} \rightarrow \infty$, the cross sections for virtual and real photons become identical, $\sigma_{\gamma^{*}}\left(W^{2}, Q^{2}\right) / \sigma_{\gamma p}\left(W^{2}\right) \rightarrow 1$. The gluon density deduced from the colour-dipole cross section fulfills the leading order DGLAP relationship. Evolution à la DGLAP breaks down for $\eta \lesssim 0.1$.
\end{abstract}

* Supported by the BMBF, Contract 05 HT9PBA2

** Permanent address

*** On leave from BLTP JINR, Dubna, Russia 
Recently we have shown that the data for the total virtual-photon-proton cross section in the low-x diffraction region $\left(x \simeq \frac{Q^{2}}{W^{2}} \ll 1\right)$ fulfill a scaling law. We found [1]

$$
\sigma_{\gamma^{*} p}\left(W^{2}, Q^{2}\right)=\sigma_{\gamma^{*} p}\left(\eta\left(W^{2}, Q^{2}\right)\right)
$$

where the dimensionless scaling variable $\eta$ is given by

$$
\eta\left(W^{2}, Q^{2}\right)=\frac{Q^{2}+m_{0}^{2}}{\Lambda^{2}\left(W^{2}\right)},
$$

and $\Lambda^{2}\left(W^{2}\right)$ is a (slowly) increasing function of $W^{2}$, conveniently parameterized by the power law

$$
\Lambda^{2}\left(W^{2}\right)=C_{1}\left(W^{2}+W_{0}^{2}\right)^{C_{2}}
$$

or by a logarithmic function of $W^{2}$. The scaling behaviour (11) was determined in a model-independent analysis of the experimental data and subsequently it was interpreted [1] in terms of an ansatz [2] for the virtual-photon-proton cross section that was based on the generic structure of two-gluon exchange [3, 4] (compare Fig.1), the generalized vector dominance/colour-dipole picture (GVD/CDP). In the model-independent analysis of the data no specific ansatz for the functional dependence of the cross section on $\eta$ (apart from continuity in $\eta$ ) was introduced. The threshold mass, $m_{0}<m_{\rho^{0}}$, as well as the parameters $W_{0}^{2}$ and $C_{2}$ У, were determined in a least-squares fit to the experimental data. The theoretical ansatz for $\sigma_{\gamma^{*} p}$, or rather the virtual-photon-proton Compton-forward-scattering-amplitude in terms of (the generic structure of) two-gluon exchange, the GVD/CDP, was shown to incorporate the observed scaling, and a comparison with the experimental data determined the fourth parameter $C_{2}$.

The purpose of the present work is threefold,

i) to elaborate on the consequences of the observed scaling in $\eta$ with respect to the asymptotic behaviour of $\sigma_{\gamma^{*} p}$ in the so-called "saturation" regime, $Q^{2}$ fixed, $W^{2} \rightarrow \infty$, i.e. $x \rightarrow 0$,

ii) to include the more recently published data from ZEUS and H1 in the analysis, and

\footnotetext{
${ }^{1}$ The absolute value of $C_{1}>0$ is irrelevant for the existence of the scaling behaviour. It only corresponds to a shift of the scale.
} 
iii) to establish the connection between the above-mentioned description based on the notion of the colour-dipole cross section and the conventional gluon density of the proton.

The evaluation of the two-gluon exchange diagrams from Fig.1 in the low- $x$ limit, and transition to transverse position space, leads to [3, 1]

$$
\begin{aligned}
\sigma_{\gamma^{*} p}\left(W^{2}, Q^{2}\right)= & \int d z \int d^{2} r_{\perp}|\psi|^{2}\left(r_{\perp}^{2} Q^{2} z(1-z), Q^{2} z(1-z), z\right) \cdot \\
\cdot & \sigma_{(q \bar{q}) p}\left(r_{\perp}^{2}, z(1-z), W^{2}\right) .
\end{aligned}
$$

where the Fourier representation of the colour-dipole cross section is given by

$$
\sigma_{(q \bar{q}) p}\left(r_{\perp}^{2}, z(1-z), W^{2}\right)=\int d^{2} l_{\perp} \tilde{\sigma}_{(q \bar{q}) p}\left(\vec{l}_{\perp}^{2}, z(1-z), W^{2}\right) \cdot\left(1-e^{-i \vec{l}_{\perp} \cdot \vec{r}_{\perp}}\right)
$$

Indeed, substituting (5) into (4), together with the Fourier representation of the photon-wave function $|\psi|^{2}$, yields [2] precisely the momentum-space representation for the cross section based on the diagrams in Fig.1 evaluated in the $x \rightarrow 0$ limit. We note that the detailed structure of the interaction of the $(q \bar{q})$ pair, once the pair has emitted (at least) two gluons, is fully buried in the colour-dipole cross section, or rather in its Fourier-transform, $\tilde{\sigma}_{(q \bar{q}) p}$, in (5).

From (5), for vanishing and for infinite quark-antiquark separation, respectively, we have

$$
\sigma_{(q \bar{q}) p}\left(r_{\perp}^{2}, z(1-z), W^{2}\right)=\sigma^{(\infty)} \cdot \begin{cases}\frac{1}{4} r_{\perp}^{2}\left\langle\vec{l}^{2}\right\rangle_{W^{2}, z} & , \quad \text { for } r_{\perp}^{2} \rightarrow 0 \\ 1 & , \text { for } r_{\perp}^{2} \rightarrow \infty\end{cases}
$$

where by definition

$$
\sigma^{(\infty)}=\pi \int d l_{\perp}^{2} \tilde{\sigma}\left(\vec{l}_{\perp}^{2}, z(1-z), W^{2}\right)
$$

and $\left\langle\vec{l}_{\perp}^{2}\right\rangle$, the average gluon transverse momentum, is given by $[5]$

$$
\left\langle\vec{l}^{2}\right\rangle_{W^{2}, z}=\frac{\int d \vec{l}_{\perp}^{2} \vec{l}_{\perp}^{2} \tilde{\sigma}_{(q \bar{q}) p}\left(\vec{l}_{\perp}^{2}, z(1-z), W^{2}\right)}{\int d \vec{l}_{\perp}^{2} \tilde{\sigma}_{(q \bar{q}) p}\left(\vec{l}_{\perp}^{2}, z(1-z), W^{2}\right)}
$$

If the GVD/CDP defined by (4) and (5) is to be consistent, the asymptotic $\left(\vec{r}_{\perp}^{2} \rightarrow \infty\right)$ limit of the colour-dipole cross section, $\sigma^{(\infty)}$, defined in (17), must 
exist, and moreover it should be independent of the configuration variable $z$. In addition, $\sigma^{(\infty)}$ should at most be weakly dependent on $W^{2}$, to fulfill the unitarity restrictions for hadron-hadron interactions for $W^{2} \rightarrow \infty$.

In order to incorporate the requirement of scaling, (1), we replace $\vec{r}_{\perp}^{2}$ in (田) by the dimensionless variable [5]

$$
u \equiv r_{\perp}^{2} \Lambda^{2}\left(W^{2}\right) z(1-z)
$$

The photon wave function in (4) then becomes the function $|\psi|^{2}\left(u \frac{Q^{2}}{\Lambda^{2}}, \frac{Q^{2}}{\Lambda^{2}}, z\right)$. Scaling in $\eta$ implies that the colour-dipole cross section solely depends on $u$,

$$
\sigma_{(q \bar{q}) p}\left(r_{\perp}^{2}, z(1-z), W^{2}\right)=\sigma_{(q \bar{q}) p}(u)
$$

and according to (6), we have

$$
\left\langle\vec{l}^{2}\right\rangle_{W^{2}, z}=\Lambda^{2}\left(W^{2}\right) z(1-z)
$$

Averaging over the configuration variable $z$ yields

$$
\left\langle\vec{l}^{2}\right\rangle_{W^{2}}=\frac{1}{6} \Lambda^{2}\left(W^{2}\right)
$$

The quantity $\Lambda^{2}\left(W^{2}\right)$ appearing in the scaling variable (2) is accordingly (apart from the factor $1 / 6$ ) identified as the average transverse momentum of the gluon absorbed by the quark (or the antiquark).

It is illuminating to explicitly insert the average gluon transverse momentum (11) into (6), yielding

$$
\sigma_{q \bar{q} p}=\sigma^{(\infty)} \cdot \begin{cases}\frac{1}{4} r_{\perp}^{2} \Lambda^{2}\left(W^{2}\right) z(1-z) & , \text { for } \Lambda^{2} \cdot r_{\perp}^{2} \rightarrow 0 \\ 1 & , \text { for } \Lambda^{2} \cdot r_{\perp}^{2} \rightarrow \infty\end{cases}
$$

Noting that the dependence of the photon wave function on $\vec{r}_{\perp}^{2} \cdot Q^{2}$ requires small $\vec{r}_{\perp}^{2}$ at large $Q^{2}$ in order to develop appreciable strength, an important qualitative conclusion on the functional dependence on $\eta$ of $\sigma_{\gamma^{*} p}(\eta)$ can immediately be drawn. For fixed energy and sufficiently large $Q^{2}$, the dominant contribution to $\sigma_{\gamma^{*} p}(\eta)$ stems from the $\Lambda^{2} \cdot \vec{r}_{\perp}^{2} \rightarrow 0$ behaviour of the dipole cross section in (13). Increasing the energy $W^{2}$ and, accordingly, $\Lambda^{2}\left(W^{2}\right)$ at fixed $Q^{2}$, the $\Lambda^{2} \cdot r_{\perp}^{2} \rightarrow \infty$ 
behaviour of the dipole cross section in (13) will eventually become relevant, thus implying the transition from a fairly strong $W^{2}$ dependence (due to $\Lambda^{2}\left(W^{2}\right)$ ) to an increasingly weaker one. This behaviour is precisely borne out by the data: The strong dependence on $W^{2}$ at fixed $Q^{2}$ indeed turns into the weaker one of photoproduction, compare Fig.2. Thus, having supplemented the GVD/CDP, (4),(5), by scaling in $\eta$, we qualitatively obtain the functional behaviour of the experimental data in Fig.2.

For the quantitative evaluation of (4) with (5), we approximated the (unknown) precise distribution in the gluon momentum transfer $\vec{l}_{\perp}^{2}$ by a $\delta$-function situated at the average gluon momentum (11), i.e. [1]

$$
\tilde{\sigma}_{(q \bar{q}) p}=\sigma^{(\infty)} \frac{1}{\pi} \delta\left(\vec{l}_{\perp}^{2}-\Lambda^{2}\left(W^{2}\right) z(1-z)\right) .
$$

With (14), and upon returning to momentum space, a complete analytical evaluation of (4) with (5) was given. Ignoring corrections of the order of $m_{0}^{2} / \Lambda^{2}\left(W^{2}\right) \ll$ 1 , it reads $[1]]^{2}$

$$
\sigma_{\gamma^{*} p}(\eta)=\frac{\alpha R_{e^{+} e^{-}}}{3 \pi} \sigma^{(\infty)} I_{0}(\eta) \cong \frac{2 \alpha}{3 \pi} \sigma^{(\infty)} \begin{cases}\ln (1 / \eta), & \text { for } \eta \rightarrow 0 \\ 1 / 2 \eta, & , \text { for } \eta \gg 1\end{cases}
$$

For any arbitrary fixed value of $Q^{2}$, with $W^{2} \rightarrow \infty$, the soft logarithmic dependence of photoproduction and hadronic reactions as a function of $\eta^{-1}=$ $\Lambda^{2}\left(W^{2}\right) /\left(Q^{2}+m_{0}^{2}\right)$ is indeed reached.

From the logarithmic dependence on $\eta$ for $\eta \rightarrow 0$ in (15), we draw the important conclusion that virtual and real photons become equivalent in the limit of $W^{2} \rightarrow \infty$ (i.e. $x \rightarrow 0, Q^{2}$ fixed)

$$
\lim _{\substack{W^{2} \rightarrow \infty \\ Q^{2} \text { fixed }}} \frac{\sigma_{\gamma^{*} p}\left(W^{2}, Q^{2}\right)}{\sigma_{\gamma p}\left(W^{2}\right)}=1 .
$$

Note that the alternative of $\Lambda^{2}=$ const. that implies Bjorken scaling of the structure function $F_{2} \sim Q^{2} \sigma_{\gamma^{*}}$ for sufficiently large $Q^{2}$, leads to a result entirely different from (16),

$$
\lim _{\substack{W^{2} \rightarrow \infty \\ Q^{2} \text { fixed }}} \frac{\sigma_{\gamma^{*} p}\left(W^{2}, Q^{2}\right)}{\sigma_{\gamma p}\left(W^{2}\right)}=\frac{\Lambda^{2}}{2 Q^{2} \ln \frac{\Lambda^{2}}{m_{0}^{2}}}, \quad(\text { assuming } \Lambda=\text { const. }),
$$

\footnotetext{
${ }^{2}$ Compare [1] for the complete expression from the function $I_{0}(\eta)$
} 
i.e. a suppression of the virtual-photon-proton cross section relative to photoproduction by a full power of $Q^{2}$ even in the asymptotic limit of $W^{2} \rightarrow \infty, Q^{2}$ fixed $(x \rightarrow 0)$.

In our reanalysis of the experimental data [6, ]], we have replaced the H1 94 and the ZEUS 94 results by the most recent H1 96/97 and ZEUS 96/97 data. The result for the fitted parameters differs only within errors from the previous fit. This can be seen by comparing our new results,

$$
\begin{aligned}
m_{0}^{2} & =0.15 \pm 0.04 \mathrm{GeV}^{2} \\
C_{1} & =0.34 \pm 0.06, \\
C_{2} & =0.27 \pm 0.01 \\
W_{0}^{2} & =1081 \pm 124 \mathrm{GeV}^{2},
\end{aligned}
$$

with the ones in [1]. Figure 2 shows the data and the results from the GVD/CDP based on the parameter set (18).

We return to the above discussion on the asymptotic behaviour of $\sigma_{\gamma^{*} p}$ given by (16). The approach to this limit of $x \rightarrow 0$ is governed by the limit of $\eta \rightarrow 0$ in Fig.2. It is suggestive to consider the logarithmic derivative of $\ln \sigma_{\gamma^{*} p}(\eta)$. From (15), we have

$$
-\frac{d}{d \ln \eta} \ln \sigma_{\gamma^{*} p}(\eta)=\frac{1}{I_{0}(\eta)} \eta \frac{d I_{0}(\eta)}{d \eta}= \begin{cases}\frac{1}{\ln \eta^{-1}}, & , \text { for } \eta \rightarrow 0, \\ 1, & , \text { for } \eta \rightarrow \infty .\end{cases}
$$

In Fig.3, we show the logarithmic derivative (19) in conjuction with the dependence of $W^{2}$ on $Q^{2}$ at fixed $\eta$. The "saturation limit" of a soft $W^{2}$ dependence ( $Q^{2}$ arbitrary, fixed) requires $\eta \leq 0.1$ The corresponding energy is extremely high, as may be read off from Fig.3b.

The approach of $\sigma_{\gamma^{*} p}$ to the limit (16) is also explicitly displayed in Fig.4 that shows $\sigma_{\gamma^{*} p}$ as a function of $W^{2}$ for various fixed values of $Q^{2}$. We stress that the limiting behaviour (16), i.e. the universal cross section for virtual and real photons, is the outgrowth of the $\ln (1 / \eta)$ behaviour in (15) for $\eta \rightarrow 0$. This logarithmic functional dependence is a strict consequence of the GVD/CDP with scaling in $\eta$ that rests on the underlying generic two-gluon-exchange structure from QCD in Fig.1. 
So far we have exclusively concentrated on a representation of $\sigma_{\gamma^{*} p}$ in terms of the colour-dipole cross section, $\sigma_{(q \bar{q}) p}\left(\vec{r}_{\perp}^{2}, W^{2}, z(1-z)\right)$. For sufficiently large $Q^{2}$ and non-asymptotic $W^{2}$, such that the $\Lambda^{2}\left(W^{2}\right) \cdot \vec{r}_{\perp}^{2} \rightarrow 0$ limit in (13) is valid, one may alternatively parameterize the lower vertex in Fig.1 in terms of the gluon density of the proton. The corresponding formula has indeed been worked out in [8]. It reads

$$
\sigma_{(q \bar{q}) p}\left(r_{\perp}^{2}, x, Q^{2}\right)=\frac{\pi^{2}}{3} r_{\perp}^{2} x g\left(x, Q^{2}\right) \alpha_{s}\left(Q^{2}\right) .
$$

Identifying (20) with the $\Lambda^{2}\left(W^{2}\right) \cdot r_{\perp}^{2} \rightarrow 0$ form of $\sigma_{(q \bar{q}) p}$ from (13), upon averaging over $z(1-z)$ as in (12),

$$
\bar{\sigma}_{(q \bar{q}) p}\left(r_{\perp}^{2}, W^{2}\right)=\sigma^{(\infty)} \frac{1}{24} r_{\perp}^{2} \Lambda^{2}\left(W^{2}\right),
$$

we deduce

$$
x g\left(x, Q^{2}\right) \alpha_{s}\left(Q^{2}\right)=\frac{1}{8 \pi^{2}} \sigma^{(\infty)} \Lambda^{2}\left(\frac{Q^{2}}{x}\right) .
$$

The functional behaviour of $\Lambda^{2}\left(W^{2}\right)=\Lambda^{2}\left(\frac{Q^{2}}{x}\right)$ responsible for the $\vec{r}_{\perp}^{2} \rightarrow 0$ dependence of the colour dipole cross section thus determines (or provides a model for) the gluon density.

One may ask the additional question whether (22) is consistent with the result obtained by assuming the validity of the DGLAP evolution [9] in application to our result for $F_{2}=\left(Q^{2} / 4 \pi^{2} \alpha\right) \sigma_{\gamma^{*} p}$. This is indeed the case. Assuming dominance of the gluon distribution at low $x$ [10], we have with 4 quark flavours contributing,

$$
\frac{\partial F_{2}\left(\frac{x}{2}, Q^{2}\right)}{\partial \ln Q^{2}}=\frac{10}{27 \pi} x g(x) \alpha_{s}\left(Q^{2}\right) .
$$

Using the $\eta \gg 1$ approximation in (15) and noting that (3) yields

$$
\frac{\partial \Lambda^{2}\left(\frac{Q^{2}}{x}\right)}{\partial \ln Q^{2}}=C_{2} \Lambda^{2}\left(\frac{Q^{2}}{x}\right),
$$

one immediately finds that (23) yields a result for the gluon density that is identical to the one in (22). In other words, the gluon density derived from the GVD/CDP fulfills the evolution equation (23). This result explicitly demonstrates the consistency of the GVD/CDP with the DGLAP approach in the limit of $\eta \gg 1$, where evolution is applicable. 
In Fig.5, we show the gluon density based on (22) [ using the evolution of $\alpha_{s}\left(Q^{2}\right)$ from the PDG [11]. For Fig.5, for $\sigma^{(\infty)}$ in (22), the value of $\sigma^{(\infty)}=$ $48 \mathrm{GeV}^{-2}=18.7 \mathrm{mb}$ was inserted, corresponding to the correct normalization of $\sigma_{\gamma^{*} p}$ for the case of four active flavours.

Having established the connection between the colour-dipole cross section of the GVD/CDP and the gluon density with evolution in $Q^{2}$, we are able to estimate the domains in the $\left(Q^{2}, x\right)$ and $\left(W^{2}, x\right)$ planes for which the evolution equation (23) breaks down. As noted, the transition from the region where the $\Lambda^{2} \cdot r_{\perp}^{2} \rightarrow 0$ approximation for the colour-dipole cross section holds, to the region where its behaviour for $\Lambda^{2} \cdot r_{\perp}^{2} \rightarrow \infty$ becomes relevant, for the photoabsorption cross section, $\sigma_{\gamma^{*}}(\eta)$, corresponds to the transition from large to small values of $\eta$. From Figs. 2 and 3, the boundary of the two regions is approximately given by $\eta \cong 0.1$. For $\eta \leq 0.1$, the connection between the gluon density and the colour-dipole cross section in (20), (22), and the evolution equation (23), breaks down. The curves in the $\left(Q^{2}, x\right)$ and $\left(W^{2}, x\right)$ planes corresponding to $\eta=0.1$ are shown in Fig.6. From Fig.6, for e.g. $Q^{2}=0.5 \mathrm{GeV}^{2}$, the breakdown of DGLAP evolution occurs for $x \leq 10^{-5}$.

In conclusion, the GVD/CDP with scaling in $\eta$ provides a unique picture for the photabsorption cross section in the diffraction region of small values of $x$, i.e. any $Q^{2}, x \rightarrow 0$. The GVD/CDP covers the kinematic domain where the concept of a gluon density evolving with $Q^{2}$ makes sense, as well as the kinematic domain, where $\sigma_{\gamma^{*} p} \sim \ln (1 / \eta)$ and the limiting behaviour of $\sigma_{\gamma^{*} p} / \sigma_{\gamma p} \rightarrow 1$ ("saturation") sets in. Scaling in $\eta$ allows for an important and fairly reliable estimate of the boundary between these two kinematic domains.

\section{Acknowledgement}

It is a pleasure to thank Wilfried Buchmüller, Leo Stodolsky and Günter Wolf for useful discussions. One of us (D.S.) thanks the theory group of the MPI für Physik in München for warm hospitality, where this work was finalized.

\footnotetext{
${ }^{3}$ We note that (20) as well as (23), and accordingly also (22), are LO QCD relations. Consequently, also the extracted gluon density in Fig.5 is a LO QCD result.
} 


\section{References}

[1] D. Schildknecht, B. Surrow, M. Tentyukov, Phys. Lett. B499, 116 (2001);

D. Schildknecht, Nucl. Phys. B Proc. Suppl. 99, 121 (2001);

G. Cvetic, D. Schildknecht, B. Surrow, M. Tentyukov, Eur. Phys. J. C 20, 77 (2001)

[2] G. Cvetic, D. Schildknecht, A. Shoshi, Eur. Phys. J. C 13, 301 (2000); Acta Physica Polonica B30, 3265 (1999); D. Schildknecht, Contribution to DIS 2000 (Liverpool, April 2000), hep-ph/0006153

[3] N.N. Nikolaev, B.G.Zakharov, Z. Phys. C 49, 607 (1991)

[4] F.E. Low, Phys. Rev. D12, 163 (1975; S. Nussinov, Phys. Rev. Lett. 34, 1286 (1975); Phys. Rev. D14, 246 (1976); J. Gunion, D. Soper, Phys. Rev. D15, 2617 (1977)

[5] D. Schildknecht, Contribution to DIS 2001, Bologna, Italy, April 27 to May 1, 2001, to appear in the DIS 2001 proceedings, hep-ph 0106188

[6] ZEUS 96/97: ZEUS Collaboration, S. Chekanov et al., DESY-01-014, submitted to Eur. Phys. J. C, M. Derrick et al., Z. f. Physik C72, 399 (1996); ZEUS SVTX 95: ZEUS Collaboration, J. Breitweg et al., Eur. Phys. J. C7, 609 (1999); ZEUS BPC 95: ZEUS Collaboration, J. Breitweg et al., Phys. Lett. B407, 432 (1997; ZEUS BPT 97: ZEUS Collaboration, J. Breitweg et al., Phys. Lett. B487, 1, 53 (2000)

[7] H1 SVTX 95: H1 Collaboration, C. Adloff et al., Nucl. Phys. B497, 3 (1997); H1 96/97: H1 Collaboration, C. Adloff et al., acdepted by Eur. Phys. J. C; H1 97: H1 Collaboration, C. Adloff et al., Eur. Phys. J. C13, 609 (2000)

[8] L. Frankfurt, A. Radyushkin, M. Strikman, Phys. Rev. D 5598 (1997)

[9] G. Altarelli and G. Parisi, Nucl. Phys. B126, 298 (1977); V.N. Gribov and L.N. Lipatov, Sov. J. Nucl. Phys. 15, 438 (1972); L.N. Lipatov, Sov. J. Nucl. Phys. 20, 96 (1975); Y.L. Dokshitzer, Sov. Phys. JETP 46, 641 (1977). 
[10] K. Prytz, Phys. Lett. B311, 286 (1993)

[11] Particle data group, Eur. Phys. J. C 3, 81 (1998) 

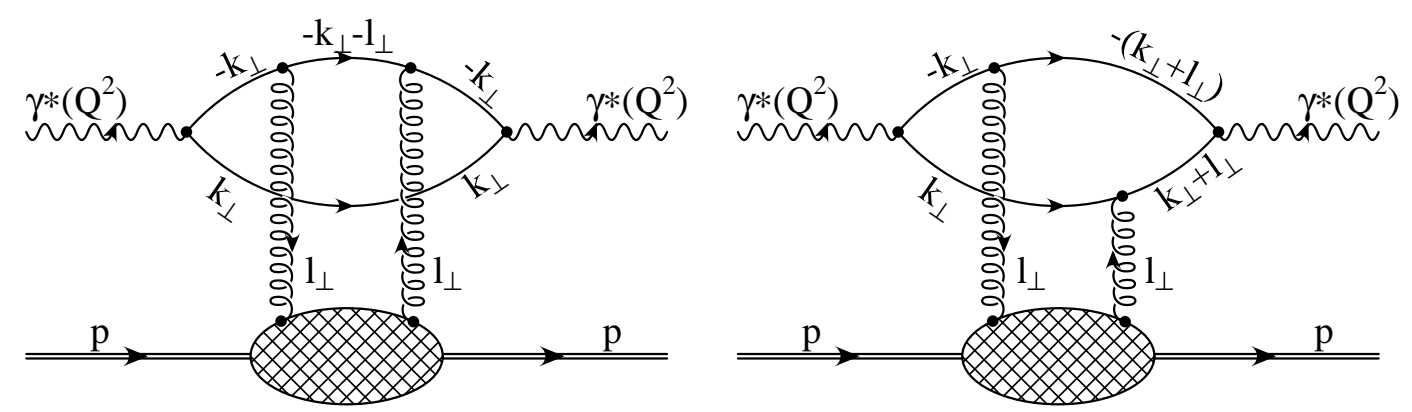

Figure 1: The two-gluon exchange. The arrows relate to the transversemomentum flow. 


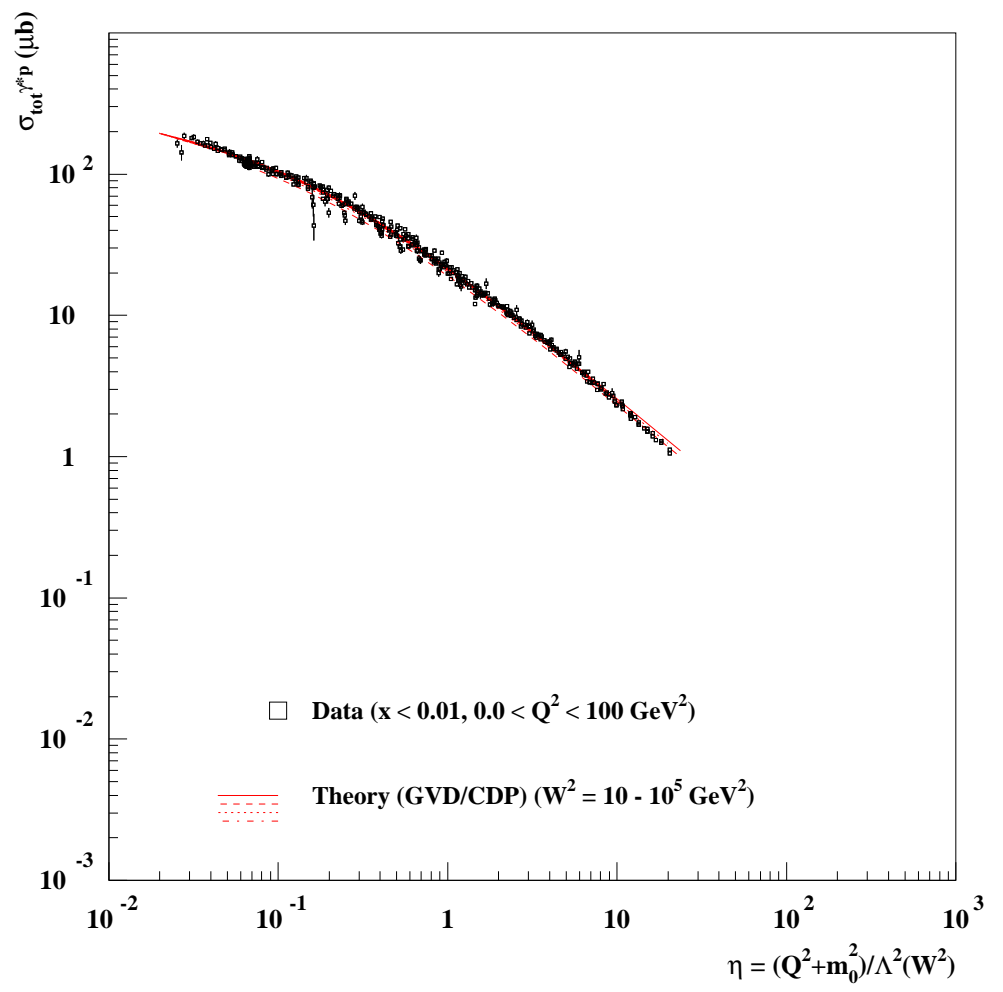

Figure 2: The virtual-photon-proton cross section, $\sigma_{\gamma^{*} p}\left(\eta\left(W^{2}, Q^{2}\right)\right)$ including $Q^{2}=0$ photoproduction as a function of $\eta=\left(Q^{2}+m_{0}^{2}\right) / \Lambda^{2}\left(W^{2}\right)$. 

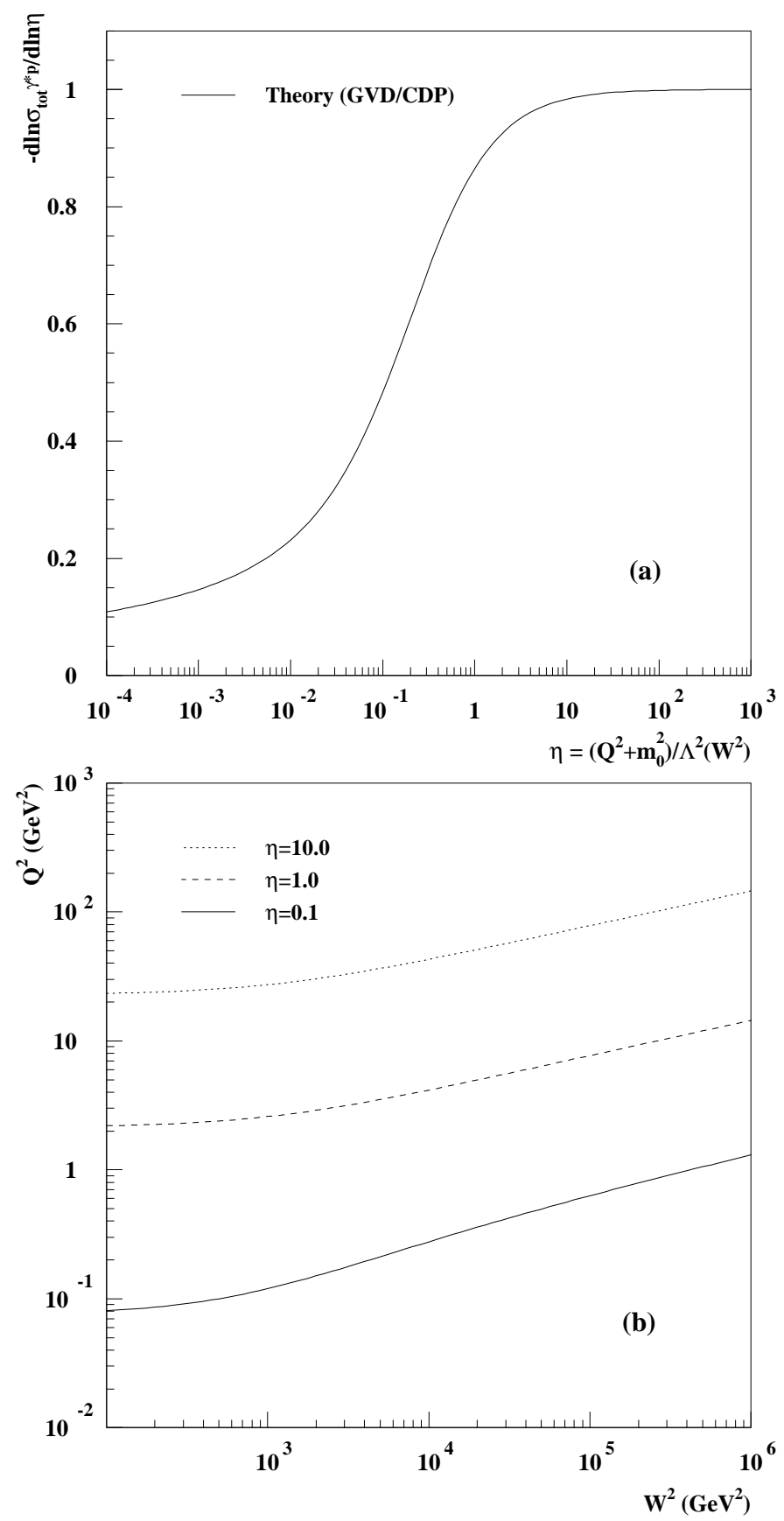

Figure 3:

a) The logarithmic derivative of $\sigma_{\gamma^{*} p}$ as a function of $\eta$.

b) The lines of $\eta=$ const in the $\left(Q^{2}, W^{2}\right)$ plane 


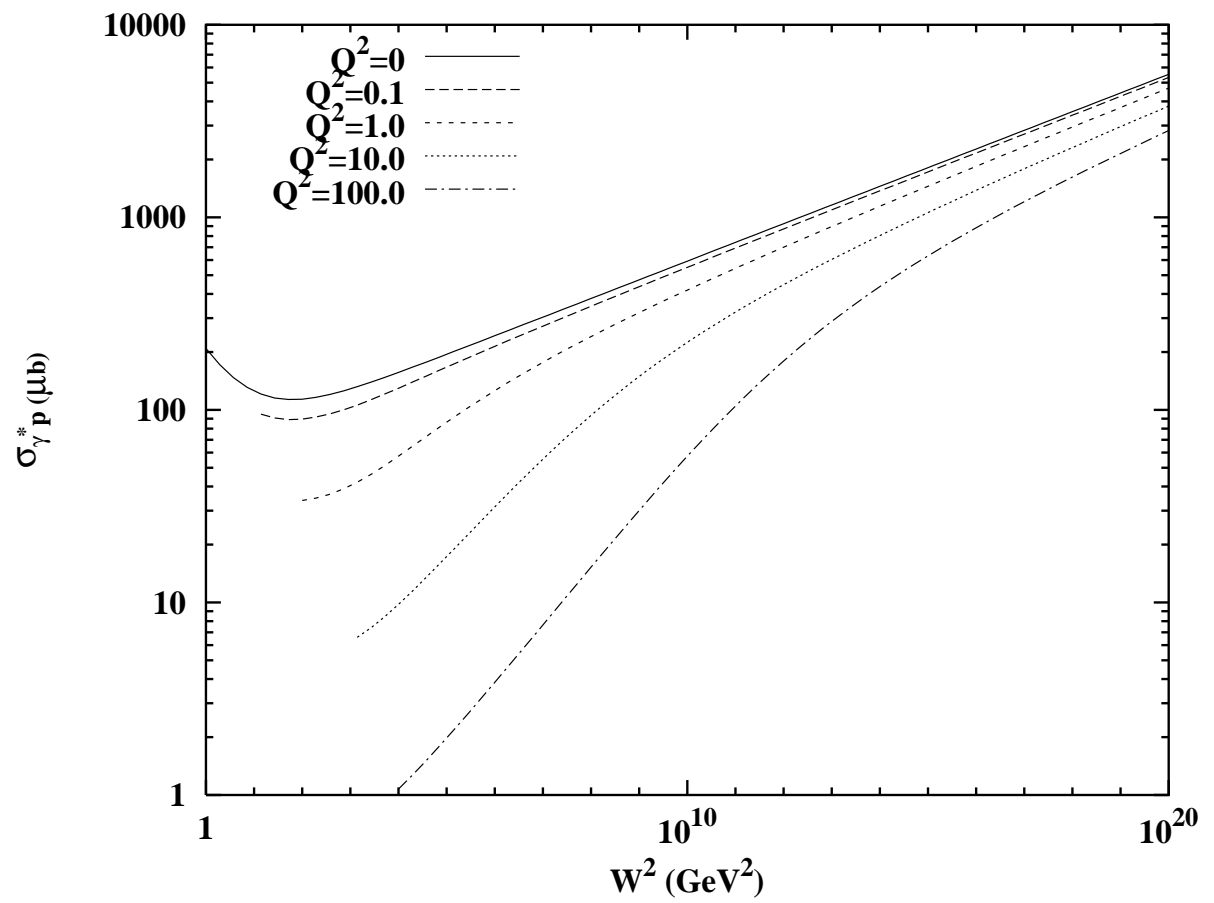

Figure 4: The virtual-photon-proton cross section, $\sigma_{\gamma^{*} p}\left(W^{2}, Q^{2}\right)$, including $Q^{2}=$ 0 photoproduction, as a function of $W^{2}$ for fixed $Q^{2}$. The figure demonstrates the asymptotic behaviour, $\sigma_{\gamma^{*} p}\left(W^{2}, Q^{2}\right) / \sigma_{\gamma p}\left(W^{2}\right) \rightarrow 1$ for $W^{2} \rightarrow \infty$. 


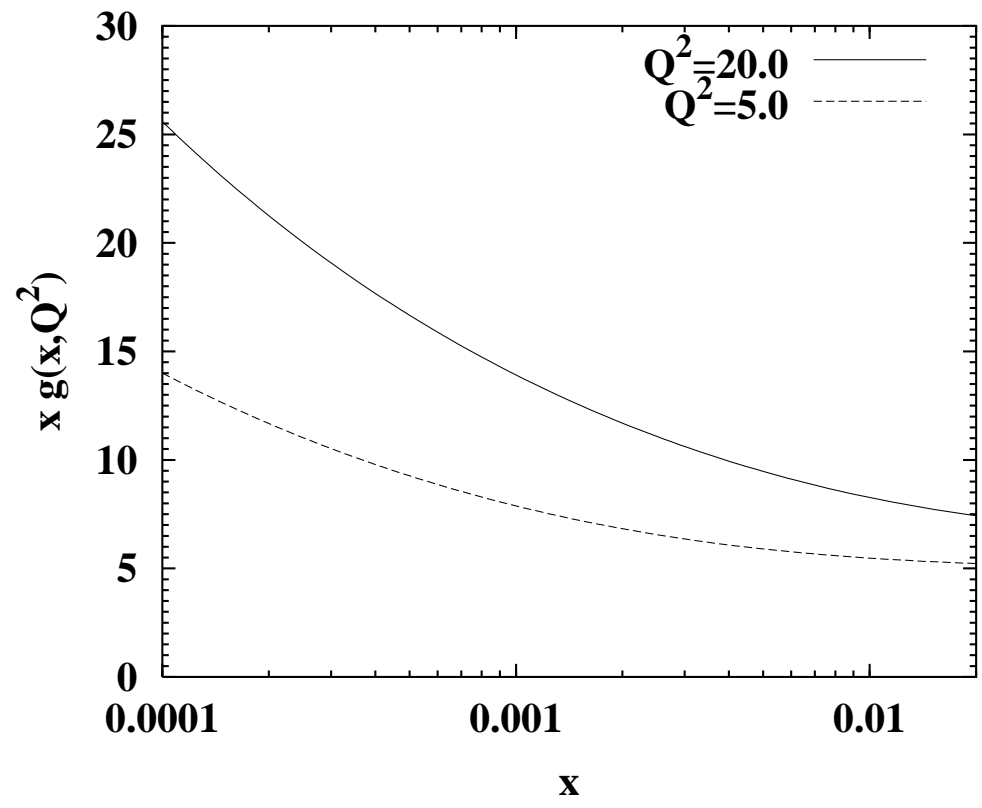

Figure 5: The gluon density corresponding to the colour-dipole cross section of the GVD/CDP. 

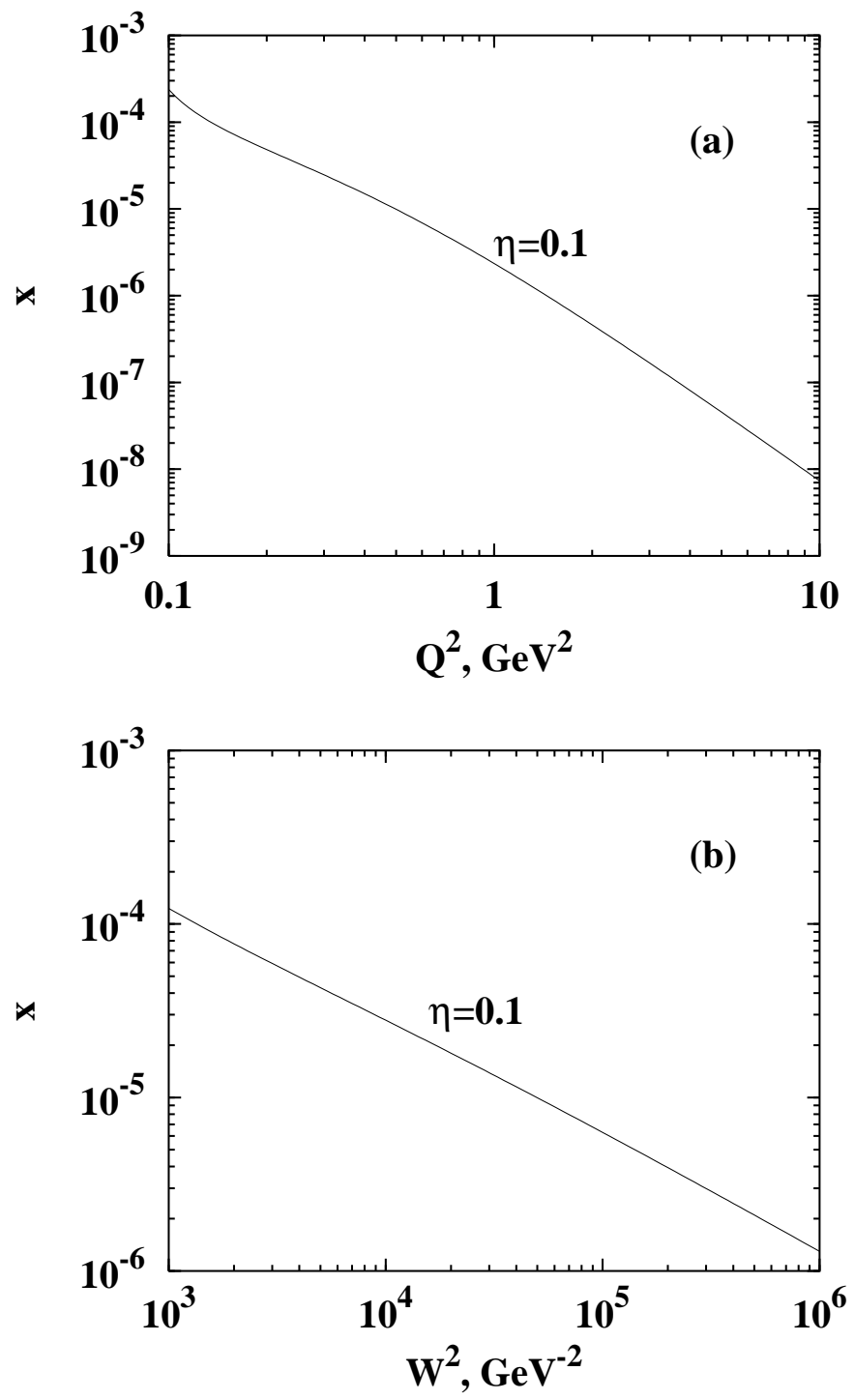

Figure 6: The lines $\eta=0.1$ in the $\left(Q^{2}, x\right)$ plane (a) and in the $\left(W^{2}, x\right)$ plane (b). The values of $x$ below the lines $\eta=0.1$ define the region, where DGLAP evolution breaks down. 